UDK 517.589, 517.926.4

T. P. Goy

Vasyl Stefanyk Precarpathian National University, Ivano-Frankivsk

\title{
Two non-elementary integral functions defined using central factorial powers
}

\begin{abstract}
We study two new real-valued non-elementary functions generated by central factorial powers. Graphs of such functions are plotted and some of their properties are proved. It is also shown that new integral functions are solutions of fourth order linear ordinary differential equations with variable coefficients.

Key words: factorial powers, central factorial powers, generalized hypergeometric function, Fresnels integrals.

Досліджено дві нові неелементарні функції дійсної змінної типу інтегралів Френеля, побудовані із застосуванням центральних факторіальних степенів. Встановлено деякі властивості цих функцій, наведено їх графіки. Виведено звичайні диференціальні рівняння, розв'язками яких є нові функції.

Ключові слова: факторіальний степінь, центральний факторіальний степінь, узагальнена гіпергеометрична функиіл, інтеграли Френеля.

Исследованы две новые неэлементарные функции действительной переменной типа интегралов Френеля, построенные при помощи центральных факториальных степеней. Установлены некоторые свойства этих функций, приведены их графики. Выведены обыкновенные дифференциальные уравнения, решениями которых являются новые функции.

Ключевые слова: факториальная степень, центральная факториальная степень, обобщенная гипергеометрическая функиия, интеграль Френеля.
\end{abstract}

\section{Introduction}

Mathematical models of many natural processes and phenomena lead to problems, exact solutions of which can not be obtained by well-known classical methods. This is a main reason for further development of numerical analysis, applied mathematics, and function theory. Extension of the "library" of non-elementary functions leads to extension the class of the problems that can be solved in closed form. Main attention is paid to the study of new non-elementary functions for further use in solving new theoretical and practical problems.

In [6] we defined new non-elementary functions $\operatorname{Sinc} x, \operatorname{Cosc} x$ constructed by replacing in a power series of trigonometric functions $\sin x, \cos x$ falling factorial powers $n^{\underline{n}}$ (i.e. usual factorials) by corresponding central powers $n^{[n]}$. Replacing in the Fresnel integrals $\int_{0}^{x} \sin t^{2} d t, \int_{0}^{x} \cos t^{2} d t$ trigonometric functions by the functions $\operatorname{Sinc} x, \operatorname{Cosc} x$ we get new real-valued functions

$$
\widehat{S}(x)=\int_{0}^{x} \operatorname{Sinc} t^{2} d t, \quad \widehat{C}(x)=\int_{0}^{x} \operatorname{Cosc} t^{2} d t .
$$


In [8] we studied similar functions constructed using rising factorial powers.

It is known that Fresnel integrals and their various generalizations appears in diffraction theory, vibration theory, design of highways and railroad tracks, robot trajectory planning, computer-aided design, technology of oil-well drilling (see, for example, $[2,4,11,12,13,16]$ and the references given there).

The aim of this paper is to study the functions $\widehat{S}(x)$ and $\widehat{C}(x)$.

\section{Preliminaries and Notations}

Definition 1. [10] For arbitrary $x \in \mathbb{R}$ and $m \in \mathbb{N}$ the expression

$$
x^{m\{k\}}=x(x+k)(x+2 k) \cdot \ldots \cdot(x+(m-1) k)
$$

is called the factorial power $m$ with the step $k \in \mathbb{R}$.

Factorial power $x^{m\{k\}}$ is called rising if $k>0$ and is called falling if $k<0$.

Definition 2. [15] For arbitrary $x \in \mathbb{R}$ and $m \in \mathbb{N}$ the expression

$$
x^{m[k]}=x\left(x+\frac{m k}{2}-k\right)\left(x+\frac{m k}{2}-2 k\right) \cdot \ldots \cdot\left(x+\frac{m k}{2}+k\right)
$$

is called the central factorial power $m$ with the step $k>0$.

By definition, put $x^{0\{k\}} \equiv 1$ and $x^{0[k]} \equiv 1$. If $k=0$, we obtain the power function, i.e. $x^{m\{0\}}=x^{m[0]}=x^{m}$.

Rising factorial powers with the step 1 (or the Pochhammer symbol) and falling factorial powers with the step $(-1)$ we will denote, respectively, by

$$
\begin{gathered}
x^{m\{1\}}=x^{\bar{m}}:=x(x+1)(x+2) \cdot \ldots \cdot(x+m-1), \\
x^{m\{-1\}}=x^{\underline{m}}:=x(x-1)(x-2) \cdot \ldots \cdot(x-m+1) .
\end{gathered}
$$

Relation between a factorial function $m$ ! and rising (falling) factorials is expressed by the formula $m !=1^{\bar{m}}=m^{\underline{m}}$.

Central factorial powers with the step 1 we will denote by $x^{[m]}$. For example,

$$
\begin{gathered}
x^{5[1]}=x^{[5]}:=\left(x-\frac{3}{2}\right)\left(x-\frac{1}{2}\right) x\left(x+\frac{1}{2}\right)\left(x+\frac{3}{2}\right), \\
x^{6[1]}=x^{[6]}:=(x-2)(x-1) x^{2}(x+1)(x+2) .
\end{gathered}
$$

As functions of $x$, the rising, falling and central factorials powers are special cases of the polynomial sequences of binomial type studied mainly in combinatorics and calculus of finite differences (see, in particular, [5], [14]).

In general, duality of factorial powers (rising, falling and central) is a common feature in the combinatorics. In other words, if a problem leads to some combinatorial identity constructed, for example, using factorial powers then often there is a dual 
combinatorial problem, which leads to a dual combinatorial identity involving rising or central factorial powers (see [9], [17]).

\section{Functions Sinc $x, \operatorname{Cosc} x$ defined by the central factorial powers}

The well-known power series

$$
\begin{gathered}
\sin x=\sum_{n=0}^{\infty} \frac{(-1)^{n}}{(2 n+1) !} x^{2 n+1}=\sum_{n=0}^{\infty} \frac{(-1)^{n}}{(2 n+1)^{2 n+1}} x^{2 n+1}, \\
\quad \cos x=\sum_{n=0}^{\infty} \frac{(-1)^{n}}{(2 n) !} x^{2 n}=\sum_{n=0}^{\infty} \frac{(-1)^{n}}{(2 n) \underline{2 n}} x^{2 n}
\end{gathered}
$$

can be treated as the series constructed using the rising factorial powers. In analogy to these series in [6] we investigate new non-elementary functions Sinc $x, \operatorname{Cosc} x$ constructed using the central factorial powers

$$
\begin{aligned}
\text { Sinc } x & =\sum_{n=0}^{\infty} \frac{(-1)^{n}}{(2 n+1)^{[2 n+1]}} x^{2 n+1}=\frac{x}{1}-\frac{x^{3}}{\frac{5}{2} \cdot 3 \cdot \frac{7}{2}}+\frac{x^{5}}{\frac{7}{2} \cdot \frac{9}{2} \cdot 5 \cdot \frac{11}{2} \cdot \frac{13}{2}}-\ldots, \\
\operatorname{Cosc} x & =\sum_{n=0}^{\infty} \frac{(-1)^{n}}{(2 n)^{[2 n]}} x^{2 n}=1-\frac{x^{2}}{2 \cdot 2}+\frac{x^{4}}{3 \cdot 4 \cdot 4 \cdot 5}-\frac{x^{6}}{4 \cdot 5 \cdot 6 \cdot 6 \cdot 7 \cdot 8}+\ldots
\end{aligned}
$$

Clearly

$$
\begin{gathered}
\text { Sinc } x=x+\sum_{n=1}^{\infty} \frac{(-1)^{n} 4^{n}(2 n-1) ! !}{(6 n+1) ! !} x^{2 n+1}, \\
\operatorname{Cosc} x=1+\sum_{n=1}^{\infty} \frac{(-1)^{n}(n-1) !}{2(3 n-1) !} x^{2 n} .
\end{gathered}
$$

Absolute convergence on the real axis of the series (1) and (2) can easily be shown.

Let ${ }_{p} F_{q}\left(a_{1}, \ldots, a_{p} ; b_{1}, \ldots, b_{q} ; z\right)$ be a generalized hypergeometric function defined by generalized hypergeometric series, i.e. [3]

$$
{ }_{p} F_{q}\left(a_{1}, \ldots, a_{p} ; b_{1}, \ldots, b_{q} ; z\right)=\sum_{n=0}^{\infty} \frac{a_{1}^{\bar{n}} a_{2}^{\bar{n}} \cdot \ldots \cdot a_{p}^{\bar{n}}}{b_{1}^{\bar{n}} b_{2}^{\bar{n}} \cdot \ldots \cdot b_{q}^{\bar{n}}} \cdot \frac{z^{n}}{n !},
$$

where $a^{\bar{n}}$ is a rising factorial power with the step 1 .

Proposition 1. For all real $x$ the equalities

$$
\begin{gathered}
\text { Sinc } x=x \cdot{ }_{1} F_{2}\left(1 ; \frac{5}{6}, \frac{7}{6} ;-\frac{x^{2}}{27}\right), \\
\operatorname{Cosc} x=1-\frac{x^{2}}{4} \cdot{ }_{1} F_{2}\left(1 ; \frac{4}{3}, \frac{5}{3} ;-\frac{x^{2}}{27}\right)
\end{gathered}
$$

are valid. 
Proof. First we prove formula (4). With $(1)$ given that $(2 s+1) ! !=\frac{(2 s+1) !}{2^{s} s !}$ we obtain

$$
\begin{aligned}
& \text { Sinc } x=x-32 x^{3} \sum_{n=0}^{\infty} \frac{(-1)^{n} 16^{n}(2 n+1) !(3 n+3) !}{n !(6 n+7) !} x^{2 n}= \\
& =x\left(1+\sum_{n=1}^{\infty} \frac{1}{\left(\frac{5}{6} \cdot \frac{11}{6} \cdot \ldots \cdot \frac{6 n-1}{6}\right)\left(\frac{7}{6} \cdot \frac{13}{6} \cdot \ldots \cdot \frac{6 n+1}{6}\right)}\left(-\frac{x^{2}}{27}\right)^{n}\right)= \\
& =x \sum_{n=0}^{\infty} \frac{1^{\bar{n}}}{\left(\frac{5}{6}\right)^{\bar{n}}\left(\frac{7}{6}\right)^{\bar{n}} n !}\left(-\frac{x^{2}}{27}\right)^{n}=x \cdot{ }_{1} F_{2}\left(1 ; \frac{5}{6}, \frac{7}{6} ;-\frac{x^{2}}{27}\right) .
\end{aligned}
$$

Similarly, with (2) we obtain that the function Cosc $x$ can be written as

$$
\begin{aligned}
& \operatorname{Cosc} x=1-\frac{x^{2}}{4}\left(1+\sum_{n=1}^{\infty} \frac{1}{\left(\frac{4}{3} \cdot \frac{7}{3} \cdot \ldots \cdot \frac{3 n+1}{3}\right)\left(\frac{5}{3} \cdot \frac{8}{3} \cdot \ldots \cdot \frac{3 n+2}{3}\right)}\left(-\frac{x^{2}}{27}\right)^{n}\right)= \\
& =1-\frac{x^{2}}{4} \sum_{n=0}^{\infty} \frac{1^{\bar{n}}}{\left(\frac{4}{3}\right)^{\bar{n}}\left(\frac{5}{3}\right)^{\bar{n}} n !}\left(-\frac{x^{2}}{27}\right)^{n}=1-\frac{x^{2}}{4} \cdot{ }_{1} F_{2}\left(1 ; \frac{4}{3}, \frac{5}{3} ;-\frac{x^{2}}{27}\right) .
\end{aligned}
$$

Figures 1, 2 shows the graphs of the functions $y=\operatorname{Sinc} x$ and $y=\operatorname{Cosc} x$.

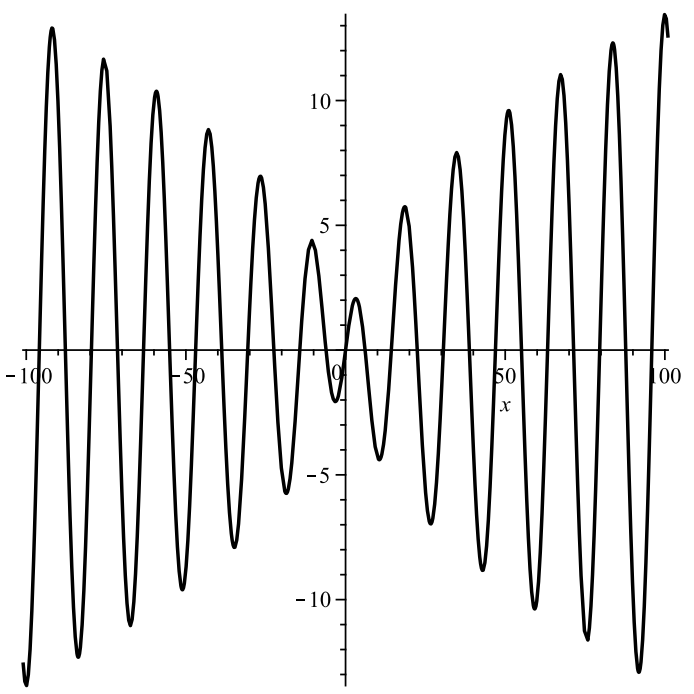

Fig. 1. Graph of the function $y=\operatorname{Sinc} x$ 


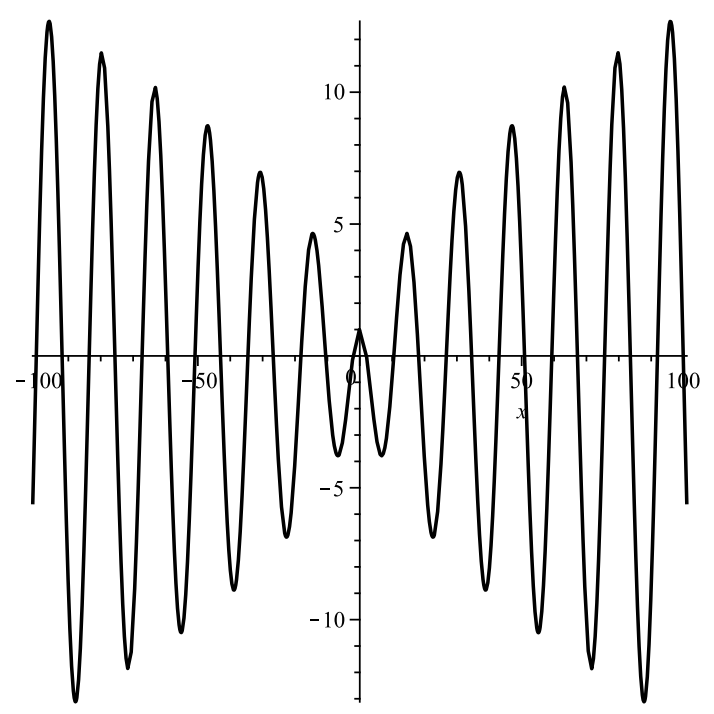

Fig. 2. Graph of the function $y=\operatorname{Cosc} x$

\section{Integral functions $\widehat{C}(x), \widehat{S}(x)$}

We will denote by $\widehat{S}(x)$ and $\widehat{C}(x)$ the integral functions defined by the formulae

$$
\begin{aligned}
& \widehat{S}(x)=\int_{0}^{x} \operatorname{Sinc} t^{2} d t, \\
& \widehat{C}(x)=\int_{0}^{x} \operatorname{Cosc} t^{2} d t .
\end{aligned}
$$

Using (1), (2), (6) and (7) we obtain the following series expansion of functions $\widehat{S}(x), \widehat{C}(x)$ :

$$
\begin{gathered}
\widehat{S}(x)=\sum_{n=0}^{\infty} \frac{(-1)^{n} 16^{n}(2 n) !(3 n) !}{n !(6 n+1) !(4 n+3)} x^{4 n+3}, \\
\widehat{C}(x)=x+\frac{1}{2} \sum_{n=1}^{\infty} \frac{(-1)^{n}(n-1) !}{(3 n-1) !(4 n+1)} x^{4 n+1} .
\end{gathered}
$$

Proposition 2. For all real $x$ the equalities

$$
\begin{gathered}
\widehat{S}(x)=\frac{x^{3}}{3} \cdot{ }_{2} F_{3}\left(\frac{3}{4}, 1 ; \frac{5}{6}, \frac{7}{6}, \frac{7}{4} ;-\frac{x^{4}}{27}\right), \\
\widehat{C}(x)=x-\frac{x^{5}}{20} \cdot{ }_{2} F_{3}\left(1, \frac{5}{4} ; \frac{4}{3}, \frac{5}{3}, \frac{9}{4} ;-\frac{x^{4}}{27}\right)
\end{gathered}
$$

are valid. 
Proof. Using (3), from (8) we obtain

$$
\begin{gathered}
\widehat{S}(x)=x^{3} \sum_{n=0}^{\infty} \frac{(-1)^{n} 16^{n}(2 n) !(3 n) !}{n !(6 n+1) !(4 n+3)} x^{4 n}= \\
=\frac{x^{3}}{3}\left(1+\sum_{n=1}^{\infty} \frac{\frac{3}{4} \cdot \frac{7}{4} \cdot \ldots \cdot \frac{4 n-1}{4}}{\left(\frac{5}{6} \cdot \frac{11}{6} \cdot \ldots \cdot \frac{6 n-1}{6}\right)\left(\frac{7}{6} \cdot \frac{13}{6} \cdot \ldots \cdot \frac{6 n+1}{6}\right)\left(\frac{7}{4} \cdot \frac{11}{4} \cdot \ldots \cdot \frac{4 n+3}{4}\right)}\left(-\frac{x^{4}}{27}\right)^{n}\right)= \\
=\frac{x^{3}}{3} \sum_{n=0}^{\infty} \frac{\left(\frac{3}{4}\right)^{\bar{n}} 1^{\bar{n}}}{\left(\frac{5}{6}\right)^{\bar{n}}\left(\frac{7}{6}\right)^{\bar{n}}\left(\frac{7}{4}\right)^{\bar{n}} n !}\left(-\frac{x^{4}}{27}\right)^{n}= \\
=\frac{x^{3}}{3} \cdot{ }_{2} F_{3}\left(\frac{3}{4}, 1 ; \frac{5}{6}, \frac{7}{6}, \frac{7}{4} ;-\frac{x^{4}}{27}\right) .
\end{gathered}
$$

Similarly, from (9), using (3), we obtain for other function:

$$
\begin{gathered}
\widehat{C}(x)=x-\frac{x^{5}}{2} \sum_{n=0}^{\infty} \frac{(-1)^{n} n !}{(3 n+2) !(4 n+5)} x^{4 n}= \\
=x-\frac{x^{5}}{20}\left(1+\sum_{n=1}^{\infty} \frac{\frac{5}{4} \cdot \frac{9}{4} \cdot \ldots \cdot \frac{4 n+1}{4}}{\left(\frac{4}{3} \cdot \frac{7}{3} \cdot \ldots \cdot \frac{3 n+1}{3}\right)\left(\frac{5}{3} \cdot \frac{8}{3} \cdot \ldots \cdot \frac{3 n+2}{3}\right)\left(\frac{9}{4} \cdot \frac{13}{4} \cdot \ldots \cdot \frac{4 n+1}{4}\right)}\left(-\frac{x^{4}}{27}\right)^{n}\right)= \\
=x-\frac{x^{5}}{20} \sum_{n=0}^{\infty} \frac{(1)^{\bar{n}}\left(\frac{5}{4}\right)^{\bar{n}}}{\left(\frac{4}{3}\right)^{\bar{n}}\left(\frac{5}{3}\right)^{\bar{n}}\left(\frac{9}{4}\right)^{\bar{n}} n !}\left(-\frac{x^{4}}{27}\right)^{n}= \\
=x-\frac{x^{5}}{20} \cdot{ }_{2} F_{3}\left(1, \frac{5}{4} ; \frac{4}{3}, \frac{5}{3}, \frac{9}{4} ;-\frac{x^{4}}{27}\right) .
\end{gathered}
$$

The graphs of functions $y=\widehat{S}(x)$ and $y=\widehat{C}(x)$ are plotted in the figures 3 and 4 .

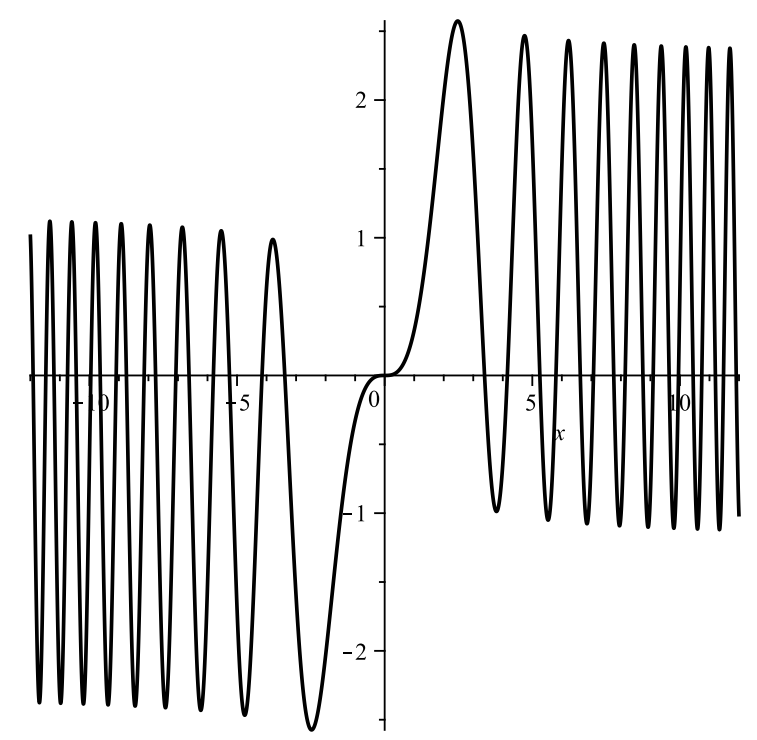

Fig. 3. Graph of the function $y=\widehat{S}(x)$ 


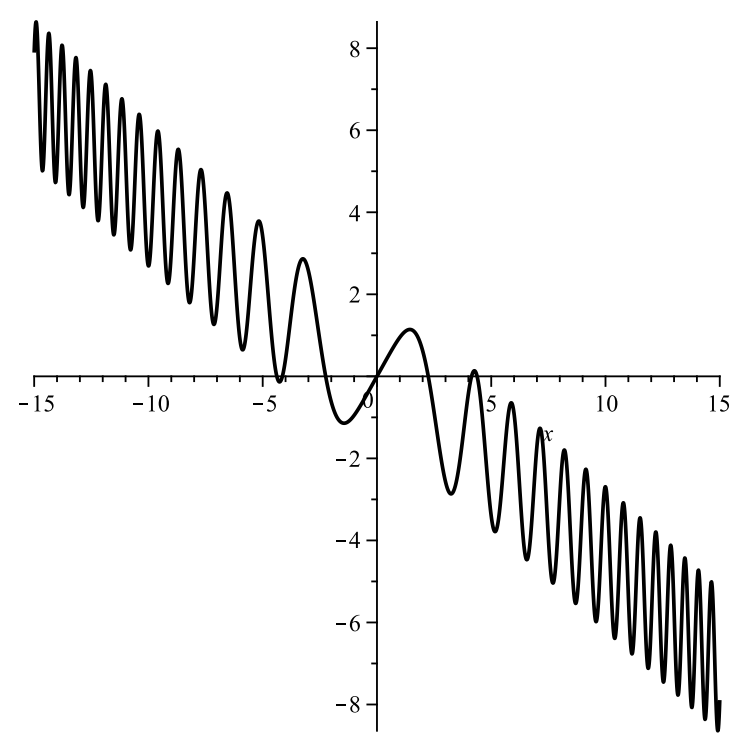

Fig. 4. Graph of the function $y=\widehat{C}(x)$

\section{Differential equations for the functions $\widehat{S}(x), \widehat{C}(x)$}

It is shown that both functions $\widehat{S}(x), \widehat{C}(x)$ are solutions of Cauchy problems for linear ordinary differential equations with polynomial coefficients.

Theorem 1. The functions $\widehat{S}(x), \widehat{C}(x)$ are solutions, respectively, of the Cauchy problems

$$
\begin{gathered}
27 x^{3} y^{(4)}-81 x^{2} y^{\prime \prime \prime}+\left(16 x^{5}+177 x\right) y^{\prime \prime}+\left(32 x^{4}-192\right) y^{\prime}=0, \\
y(0)=y^{\prime}(0)=y^{\prime \prime}(0)=0, \quad y^{\prime \prime \prime}(0)=2 ; \\
27 x^{3} y^{(4)}-135 x^{2} y^{\prime \prime \prime}+\left(16 x^{5}+339\right) y^{\prime \prime}-384 y^{\prime}=-384, \\
y(0)=0, \quad y^{\prime}(0)=1, \quad y^{\prime \prime}(0)=y^{\prime \prime \prime}(0)=0 .
\end{gathered}
$$

Proof. Using (8), (9), we obtain that the functions $\widehat{S}(x), \widehat{C}(x)$ satisfy the corresponding initial conditions from (12) or (13). It remains to check that these functions are solutions of the differential equations from (12), (13).

According to $(10)$, function $\widehat{S}(x)$ is expressed through a generalized hypergeometric function of a form ${ }_{2} F_{3}\left(a_{1}, a_{2} ; b_{1}, b_{2}, b_{3} ; z\right)$; it satisfies ordinary differential equation of the fourth order [3]

$$
\left(\sigma\left(\sigma+b_{1}-1\right)\left(\sigma+b_{2}-1\right)\left(\sigma+b_{3}-1\right)-z\left(\sigma+a_{1}\right)\left(\sigma+a_{2}\right)\right) w(z)=0,
$$

where $\sigma$ is differential operator $z \frac{d}{d z}$. Thus, the function

$$
w(z)={ }_{2} F_{3}\left(\frac{3}{4}, 1 ; \frac{5}{6}, \frac{7}{6}, \frac{7}{4} ; z\right)
$$


is the solution of the ordinary differential equation

$$
\left(\sigma\left(\sigma-\frac{1}{6}\right)\left(\sigma+\frac{1}{6}\right)\left(\sigma+\frac{3}{4}\right)-z\left(\sigma+\frac{3}{4}\right)(\sigma+1)\right) w(z)=0 .
$$

Since

$$
\begin{aligned}
\sigma^{1}=z \frac{d}{d z}, \quad \sigma^{2} & =z \frac{d}{d z}+z^{2} \frac{d^{2}}{d z^{2}}, \quad \sigma^{3}=z \frac{d}{d z}+3 z^{2} \frac{d^{2}}{d z^{2}}+z^{3} \frac{d^{3}}{d z^{3}}, \\
\sigma^{4} & =z \frac{d}{d z}+7 z^{2} \frac{d^{2}}{d z^{2}}+6 z^{3} \frac{d^{3}}{d z^{3}}+z^{4} \frac{d^{4}}{d z^{4}},
\end{aligned}
$$

from (15) after simple transformations we obtain that function (14) satisfies the linear differential equation

$$
z^{3} w^{(4)}+\frac{27}{2} z^{2} w^{\prime \prime \prime}+\left(\frac{83}{9} z-z^{2}\right) w^{\prime \prime}+\left(\frac{245}{144}-\frac{11}{4} z\right) w^{\prime}-\frac{3}{4} w=0 .
$$

Substituting independent variable in (16) by $z=-x^{4} / 27$ we get

$$
\begin{gathered}
w_{z}^{\prime}=-\frac{27}{4} \frac{w_{x}^{\prime}}{x^{3}}, \quad w_{z}^{\prime \prime}=\frac{729}{16} \frac{x w_{x}^{\prime \prime}-3 w_{x}^{\prime}}{x^{7}}, \quad w_{z}^{\prime \prime \prime}=-\frac{19683}{64} \frac{x^{2} w_{x}^{\prime \prime \prime}-9 x w_{x}^{\prime \prime}+21 w_{x}^{\prime}}{x^{11}} \\
w_{z}^{(4)}=\frac{531441}{256} \frac{x^{3} w_{x}^{(4)}-18 x^{2} w_{x}^{\prime \prime \prime}+111 x w_{x}^{\prime \prime}-231 w_{x}^{\prime}}{x^{15}}
\end{gathered}
$$

and function $w(x)={ }_{2} F_{3}\left(\frac{3}{4}, 1 ; \frac{5}{6}, \frac{7}{6}, \frac{7}{4} ;-\frac{x^{4}}{27}\right)$ is a solution of the linear homogeneous equation

$$
27 x^{3} w^{(4)}+243 x^{2} w^{\prime \prime \prime}+\left(16 x^{5}+420 x\right) w^{\prime \prime}+\left(128 x^{4}+60\right) w^{\prime}+192 x^{3} w=0 .
$$

Since according to $(10) w(x)=3 x^{-3} \cdot \widehat{S}(x)$ then from (17) we obtain

$$
\begin{gathered}
27 x^{3}\left(\frac{3 y^{(4)}}{x^{3}}-\frac{36 y^{\prime \prime \prime}}{x^{4}}+\frac{216 y^{\prime \prime}}{x^{5}}-\frac{720 y^{\prime}}{x^{6}}+\frac{1080 y}{x^{7}}\right)+ \\
+243 x^{2}\left(\frac{3 y^{\prime \prime \prime}}{x^{3}}-\frac{27 y^{\prime \prime}}{x^{4}}+\frac{108 y^{\prime}}{x^{5}}-\frac{180 y}{x^{6}}\right)+\left(16 x^{5}+420 x\right)\left(\frac{3 y^{\prime \prime}}{x^{2}}-\frac{18 y^{\prime}}{x^{4}}+\frac{36 y}{x^{5}}\right)+ \\
+\left(128 x^{4}+60\right)\left(\frac{3 y^{\prime}}{x^{3}}-\frac{9 y}{x^{4}}\right)+576 y=0
\end{gathered}
$$

it follows that the function $y=\widehat{S}(x)$ is a solution of the linear ordinary differential equation from (12).

Now it is proved that the function $\widehat{C}(x)$ is a solution of differential equation from (13). Similarly for the function $\widehat{S}(x)$ we see that hypergeometric function $w(z)={ }_{2} F_{3}\left(1, \frac{5}{4} ; \frac{4}{3}, \frac{5}{3}, \frac{9}{4} ; z\right)$ from (11) is a solution of the equation

$$
z^{3} w^{(4)}+\frac{33}{4} z^{2} w^{\prime \prime \prime}+\left(\frac{137}{9} z-z^{2}\right) w^{\prime \prime}+\left(5-\frac{13}{4} z\right) w^{\prime}-\frac{5}{4} w=0
$$


and the function $w(z)={ }_{2} F_{3}\left(1, \frac{5}{4} ; \frac{4}{3}, \frac{5}{3}, \frac{9}{4} ;-\frac{x^{4}}{27}\right)$ is a solution of the differential equation $27 x^{3} w^{(4)}+405 x^{2} w^{\prime \prime \prime}+\left(16 x^{5}+1554 x\right) w^{\prime \prime}+\left(160 x^{4}+1386\right) w^{\prime}+320 x^{3} w=0$.

Substituting into (18) $w(x)=20 x^{-5}(1-\widehat{C}(x))$ (according to (11)) we obtain that the function $y=\widehat{C}(x)$ is a solution of the equation from (13).

\section{References}

1. Abramowitz M. Handbook of Mathematical Functions with Formulas, Graphs, and Mathematical Tables / M. Abramowitz, I.A. Stegun. - N.Y., Dover, 1972.

2. Anokhov S. Physical approach to analytic simulation of Fresnel integrals / S. Anokhov // J. Opt. Soc. Am. A., 2007, Vol. 24, №1. - P. 197-203.

3. Bateman H. Higher Transcendental Functions. Vol. 1 / H. Bateman. - N.Y., McGrawHill, 1953.

4. Born M. Principles of Optics: Electromagnetic Theory of Propagation, Interference and Diffraction of Light / M. Born, E. Wolf. - Cambridge, Cambridge University Press, 1999.

5. Comtet L. Advanced Combinatorics: The Art of Finite and Infinite Expansion / L. Comtet. - Dordrecht (Netherlands), Reidel, 1972.

6. Goy T.P. On differential equations of functions generated by central factorial powers / T.P. Goy// Proc. of Crimea Inter. Math. Conf. (CIMC), 2013. - P. 4-5. (in Ukrainian)

7. Goy T.P. New functions defined by rising factorials, and its properties / T.P. Goy, R.A. Zatorsky // Bukovyna Math. J., 2013, Vol. 1, № 1-2. - P. 28-33. (in Ukrainian)

8. Goy T.P. New integral functions generated by rising factorials / T.P. Goy, R.A. Zatorsky // Carpath. Math. Publ., 2013, Vol. 5, № 2. - P. 217-224.

9. Graham R.L. Concrete Mathematics: A Foundation for Computer Science / R.L. Graham, D.E. Knuth, O. Patashnik. - Reading, Addison-Wesley, 1994.

10. Jordan C. Calculus of Finite Differences / C. Jordan. - N.Y., Chelsea, 1939.

11. Kelly A. Reactive nonholonomic trajectory generation via parametric optimal control the international / A. Kelly, B. Nagy // J. Robot. Res., 2003, Vol. 22, № 7-8. - P. 583-601.

12. Litvinov I.I. Fresnel integral in the complex form: its new representation, interpretation, and application in problems of wave beam diffraction / I.I. Litvinov // Phys. Wave Phenom., 2010, Vol. 18, № 4. - P. 267-276.

13. Monk K. An approximate solution for the wave energy shadow in the lee of an array of overtopping type wave energy converters / K. Monk, Q. Zou, D. Conley // Coast. Eng., 2013, Vol. 73. - P. 115-132.

14. Roman S. The Umbral Calculus / S. Roman. - New York, Academic Press, 1984.

15. Steffensen J.F. On the definition of the central factorial / J.F. Steffensen // J. Inst. Actuaries, 1933, Vol. 64, № 2. - P. 165-168.

16. Walton D.J. A controlled clothoid spline / D.J. Walton, D.S. Meek // Comput. Graphics, 2006, Vol. 29. - P. 353-363.

17. Zatorsky R.A. Factorial degrees and triangular matrices / R.A. Zatorsky, A.R. Malyarchuk //Carpath. Math. Publ., 2009, Vol. 1, № 2. - P. 161-171. (in Ukrainian)

Надійшла до редколегї 27.07.2014 